Journal of Innovative Optical Health Sciences

Vol. 10, No. 3 (2017) 1650050 (11 pages)

(C) The Author(s)

DOI: $10.1142 / \mathrm{S} 1793545816500504$

\title{
Monte Carlo and phantom study in the brain edema models
}

\author{
Yubing Liu, Hongke Wang, Yangyang Liu, \\ Weitao $\mathrm{Li}^{*}$ and Zhiyu Qian ${ }^{\dagger}$ \\ Department of Biomedical Engineering \\ Nanjing University of Aeronautics and Astronautics \\ 29 Yudao Street, Nanjing 210016, P.R. China \\ *liweitao@nuaa.edu.cn \\ †zhiyu@nuaa.edu.cn
}

Received 30 March 2016

Accepted 29 August 2016

Published 6 October 2016

\begin{abstract}
Because the brain edema has a crucial impact on morbidity and mortality, it is important to develop a noninvasive method to monitor the process of the brain edema effectively. When the brain edema occurs, the optical properties of the brain will change. The goal of this study is to access the feasibility and reliability of using noninvasive near-infrared spectroscopy (NIRS) monitoring method to measure the brain edema. Specifically, three models, including the water content changes in the cerebrospinal fluid (CSF), gray matter and white matter, were explored. Moreover, these models were numerically simulated by the Monte Carlo studies. Then, the phantom experiments were performed to investigate the light intensity which was measured at different detecting radius on the tissue surface. The results indicated that the light intensity correlated well with the conditions of the brain edema and the detecting radius. Briefly, at the detecting radius of $3.0 \mathrm{~cm}$ and $4.0 \mathrm{~cm}$, the light intensity has a high response to the change of tissue parameters and optical properties. Thus, it is possible to monitor the brain edema noninvasively by NIRS method and the light intensity is a reliable and simple parameter to assess the brain edema.
\end{abstract}

Keywords: Cerebrospinal fluid; gray matter; white matter; reduced scattering coefficient; light intensity.

\section{Introduction}

Patients usually suffer from the brain edema when they sustain the brain damage and diseases, such as cerebrovascular diseases, encephalitides and brain tumors. ${ }^{1}$ Brain edema is defined as the increase of the water content in brain parenchyma, which has a

${ }^{*}$ Corresponding author.

This is an Open Access article published by World Scientific Publishing Company. It is distributed under the terms of the Creative Commons Attribution 4.0 (CC-BY) License. Further distribution of this work is permitted, provided the original work is properly cited. 
vital impact on morbidity and mortality. ${ }^{2,3}$ Moreover, brain edema may lead to the expansion of brain volume, the increase of intracranial pressure (ICP) and the decrease of cerebral perfusion or oxygenation. ${ }^{4}$ Recently, several invasive and noninvasive methods have been used to monitor the process of the brain edema. Among them, ICP, ${ }^{5,6}$ measured by invasive method, is regarded as the golden standard procedure for the diagnosis of the brain edema. However, the invasive method is possible to cause intracranial infection and bleeding. Although $\mathrm{CT}^{7}$ and $\mathrm{MRI}^{8}$ are available for noninvasive diagnosis of the brain edema, the high cost and specific operating environment make them difficult to be utilized in the bedside and to monitor the brain edema for a long time. Furthermore, transcranial Doppler ultrasonography (TCD), ${ }^{9}$ flash visual evoked potentials (f-VEP) ${ }^{10}$ and electrical impedance tomography $(\mathrm{EIT})^{11}$ are also applied in the noninvasive diagnosis of the brain edema. However, the sensitivity of these methods is still needed to be improved.

Among the noninvasive methods, the near-infrared spectroscopy (NIRS) method has received more attention due to its safety and high sensitivity. NIRS method has been extensively carried out in the noninvasive measurement of biological tissue, including tissue type identification, ${ }^{12}$ cancer diagnosis ${ }^{13}$ and drug delivery process monitoring. ${ }^{14}$ The reflected light intensity carries the information about tissue structure and composition. One of the information is absorption which reflects the concentration of biological chromospheres, such as hemoglobin, myoglobin, fat and water. The other is the scattering property, which indicates the tissue information on the size of scatters and density. ${ }^{15-17}$ It is known that the near-infrared light in the 700-850 $\mathrm{nm}$ wavelength range is special to biological tissues, because the scattering property of the measuring tissue is more prominent than light absorption property at this wavelength range. ${ }^{18}$

When patients suffer from brain edema, the water content will increase inside or outside the brain cells. ${ }^{19}$ If the water content increases inside of the cells, the size of the cells will expand. While the water content increases outside the cells, the density of the cells will decrease. Therefore, the scattering property of brain tissue will be different due to the change of the size and density of brain cells. Moreover, the thickness of cerebrospinal fluid (CSF) also increases during brain edema. Hence, it is necessary to consider the change of the brain substance and CSF of the patients with brain edema.

Xie et al. ${ }^{18,20}$ and Dai et $a l .{ }^{21}$ have investigated traumatic brain edema (TBE) of rats in vivo. They reported that the reduced scattering coefficient $\left(\mu_{s}^{\prime}\right)$ of rats' cortex obtained using NIRS method related to brain edema. However, $\mu_{s}^{\prime}$ is hardly and complexly obtained in patient in vivo. Besides, the parameter is easily affected by the environmental light and is variable.

If the tissue scattering property changes, the reflectance light on the tissue surface will change. That is, the difference of light intensity indicates the change of brain physiological environment, which may be consistent with brain edema. This study aimed at investigating the feasibility and reliability of the noninvasively NIRS monitoring method of the brain edema.

In this study, we designed Monte Carlo simulations and phantom experiments to investigate the reflectance light intensity on the tissue surface during the process of brain edema. The water content will increase in different positions in the brain of patients with brain edema. Therefore, three models based on different positions were constructed. In each model, the reflectance light intensity was measured at different detecting radius on the tissue surface. Then, several phantom experiments were used to verify the accuracy of the Monte Carlo simulation results. Moreover, a series of combined models of the brain edema were simulated using the Monte Carlo method. By analyzing the reflectance light intensity on the tissue surface, it can be proved that the NIRS method has an acceptable sensitivity and specificity in monitoring the process of the brain edema.

\section{Materials and Methods}

\subsection{Experimental design}

A homemade photoelectric monitoring system was designed to detect the reflectance light intensity. The schematic diagram of the system is illustrated in Fig. 1. A beam of $760 \mathrm{~nm}$ light is emitted from a LED source, and two photoelectric detectors (OPT101, B\&B) are settled at the distance of $3.0 \mathrm{~cm}$ and $4.0 \mathrm{~cm}$, which is defined as detecting radius. The light source and detectors are fixed on a piece of rubber which could reduce the interference of environmental light. The signal is transported to 


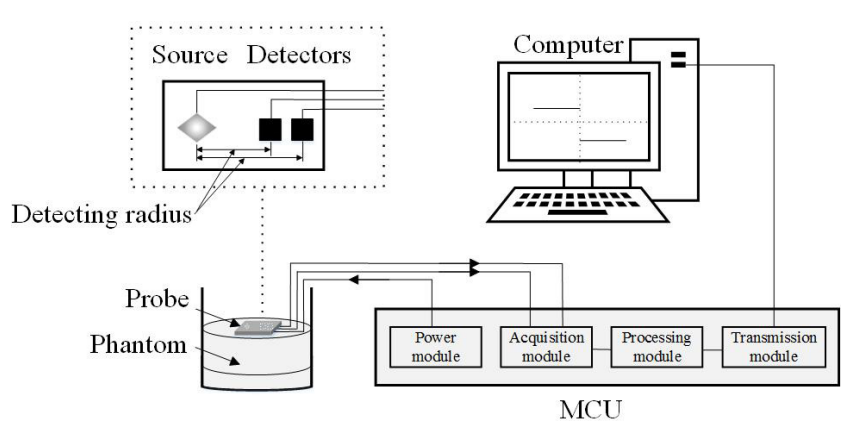

Fig. 1. The schematic diagram of the photoelectric monitoring system.

the processing module and is amplified and filtered. Then, the data is sent to a computer and displayed in the software programmed by Labview (National Instrument, USA).

\subsection{Monte Carlo simulations of brain edema}

The Monte Carlo code used in this study is written by Lihong Wang. ${ }^{22,23}$ The programs consist of Mcml program and Conv program. Mcml program simulates the single photon transmission in the tissue and Conv program is used to convolute the data from Mcml program. In the simulation, a human brain model was simplified to simulate the propagation path of light, as shown in Fig. 2. This brain model was simple and representative, which was also easy to be constructed in the phantom experiment. The tissue's optical properties of each layer are shown in Table $1,{ }^{24}$ which are measured in the same experimental condition and representative of the healthy people.

Previous studies have indicated that in the patients of brain edema, the water content will

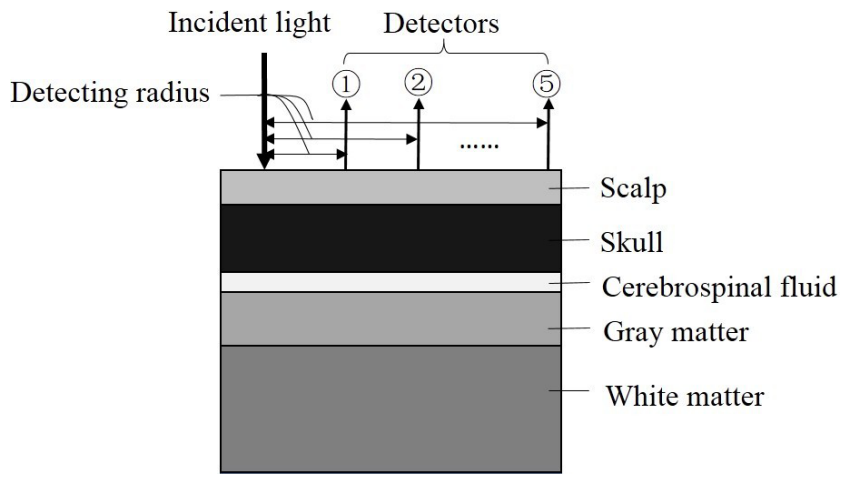

Fig. 2. The simplified Monte Carlo simulation model of human brain. mainly increase in the brain substance, including gray matter, white matter and CSF. ${ }^{25}$ Different water content will have different influence on the tissue's optical parameters of CSF, gray matter and white matter. ${ }^{26}$ In addition, the CSF, gray matter and white matter have different scattering response to the incident light. Thus, it is necessary to explore the change of reflectance light intensity for different situations of brain edema.

The absorption coefficient and the reduced scattering coefficient of biological tissue are the major parameters in the light field simulation. In the high scattering media, where $\mu_{s}^{\prime}$ is largely bigger than $\mu_{a}$, $\mu_{s}^{\prime}$ is more stable and more sensitive to the change of tissue. ${ }^{21}$ When brain edema occurs in the brain substance, the cells will become swelling and $\mu_{s}^{\prime}$ of brain tissue can reflect the change of the water content. ${ }^{20}$ Here, we mainly explored three variations, including CSF thickness, $\mu_{s}^{\prime}$ of gray matter and $\mu_{s}^{\prime}$ of white matter.

We defined three simplified models (Fig. 2) of the specific parameters to simulate the brain edema, as shown in Fig. 3. In Model 1, different thicknesses of CSF were utilized to mimic the water content change in CSF. In Model 2, different $\mu_{s}^{\prime}$ values of gray matter were set to mimic the water content change in gray matter. In Model 3 , different $\mu_{s}^{\prime}$ values of white matter were set to mimic the water content change in white matter.

The thickness of CSF of clinical patients of brain edema will be up to $1.0 \mathrm{~cm}$. Thus, in Model 1, the thickness of CSF was varying from $0 \mathrm{~cm}$ to $1.0 \mathrm{~cm}$ at $0.2 \mathrm{~cm}$ increments. Previous studies ${ }^{27}$ have introduced that the varying cell size of swelling tissue will cause different change of the scattering coefficient. Here, we used $\mu_{s}^{\prime}$ value of the healthy person, because nobody has measured the exact $\mu_{s}^{\prime}$ value of gray matter and the white matter in vivo who is suffering brain edema. Then we referred to the rat experiment results whose change region of $\mu_{s}^{\prime}$ is up to $5 \mathrm{~cm}^{-1}$, and extended the range of $\mu_{s}^{\prime}$ of gray matter into $30 \mathrm{~cm}^{-1}$ at $5 \mathrm{~cm}^{-1}$ increments and extended that of the white matter into $120 \mathrm{~cm}^{-1}$ at $20 \mathrm{~cm}^{-1}$ increments. Specifically, Model 2 simulated seven samples and the $\mu_{s}^{\prime}$ values of gray matter $[5,10,15,20,25,30,35] \mathrm{cm}^{-1}$. Model 3 simulated seven samples and $\mu_{s}^{\prime}$ values of white matter [20, 40, $60,80,100,120,140] \mathrm{cm}^{-1}$.

In the Monte Carlo simulation, the input number of photons was 1000000, the index of refraction $(n)$ for all layers was chosen to be 1.4 and the mean 
Table 1. The thickness and optical properties of the human adult brain.

\begin{tabular}{lcccc}
\hline Tissue & Thickness $(\mathrm{cm})$ & $g$ & $\begin{array}{c}\text { Absorption coefficient } \mu_{a} \\
840 \mathrm{~nm}\left(\mathrm{~cm}^{-1}\right)\end{array}$ & $\begin{array}{c}\text { Reduced scattering coefficient } \mu_{s}^{\prime} \\
840 \mathrm{~nm}\left(\mathrm{~cm}^{-1}\right)\end{array}$ \\
\hline Scalp & 0.3 & 0.9 & 0.21 & 18.1 \\
Skull & 0.7 & 0.9 & 0.19 & 15.2 \\
CSF & 0.2 & 0.9 & 0.05 & 2.3 \\
Gray matter & 0.4 & 0.9 & 0.42 & 20.9 \\
White matter & 3.4 & 0.9 & 0.17 & 86.5 \\
\hline
\end{tabular}

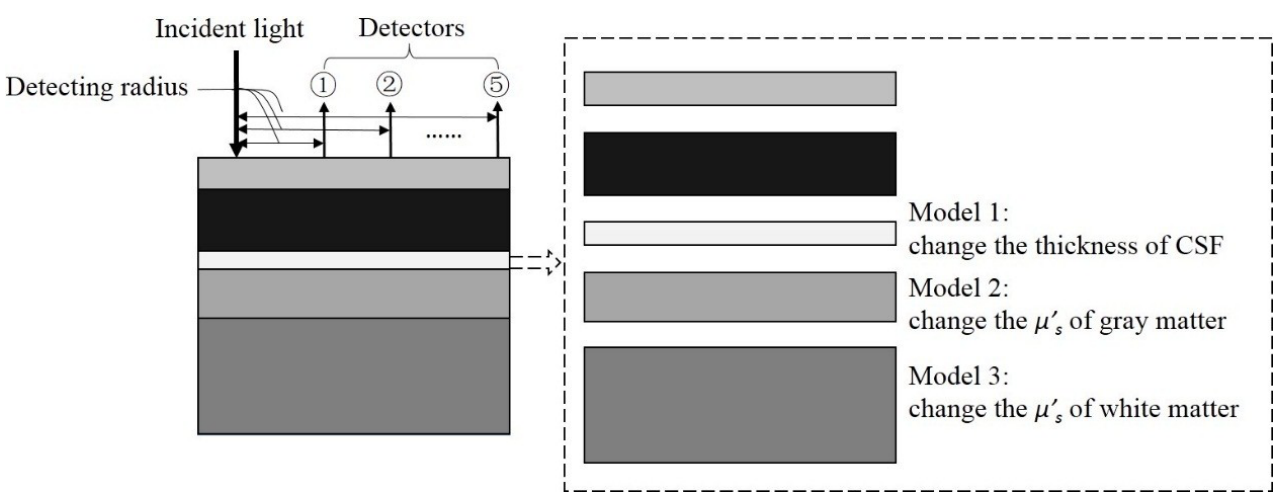

Fig. 3. Simulation model of brain edema.

cosine of scattering angle $(g)$ was 0.9 . The power of input light was $1 \mathrm{~J}$ and the radius of light beam was $0.01 \mathrm{~cm}$. The horizontal and vertical size of grid unit were $0.05 \mathrm{~cm}$ and $0.01 \mathrm{~cm}$, and the numbers of horizontal and vertical grid unit were 100 and 500 . And we finally obtained the reflectance light intensity at the detecting radius of $[1.0,2.0,3.0,4.0$, $5.0 \mathrm{~cm}$ on the tissue surface by Matlab routines.

\subsection{Phantom preparation}

In the human brain model, the thickness of scalp is $0.3 \mathrm{~cm}$. When brain edema develops, scalp has little contribution to the change of the light intensity. Thus, in the phantom experiment, only four-layer phantoms are constructed, including a porcine spealbone, water layer and a two-layer phantom. The porcine spealbone which was $0.3 \mathrm{~cm}$ thick mimic the human brain skull. The water layer was used as the CSF and a two-layer phantom was constructed as the gray matter and the white matter.

The phantom consists of gelatin powder (Sigma, St Louis, MO, USA) and pure milk. The specific concentrations of gelatin powder and milk result in different scattering properties of phantoms. ${ }^{28}$ In each phantom, $\mu_{s}^{\prime}$ values of each layer were calibrated by the ISS oximeter (96208, ISS, Champaign, IL). Figure 4 shows the process of constructing a two-layer phantom. In Fig. 4, process $\mathrm{A}$ and $\mathrm{B}$ represent the main steps of constructing the one-layer phantom and the two-layer phantom, respectively. When constructing the single layer phantom, the specific weight of the gelatin powder was added into $200 \mathrm{~mL}$ of boiling water and dissolved completely by stirring for $10 \mathrm{~min}$. After the solution cooled down, $30 \mathrm{~mL}$ of milk was poured into it and stirred for $5 \mathrm{~min}$. Next, the solution was placed in a refrigerator for $5 \mathrm{~h}$. After two one-layer phantoms were made, the top phantom was melted into solution and it was poured on the solid bottom phantom, the thickness of top solution was set to $0.4 \mathrm{~cm}$. Then the phantom was put in the refrigerator for another $5 \mathrm{~h}$. Finally, the phantom with two layers was completed.

According to the Monte Carlo simulations, the thicknesses of the water layer in Model 1 were specified in the range $[0.2,0.4,0.6,0.8,1.0] \mathrm{cm}$. In Model $2, \mu_{s}^{\prime}$ values of top phantom were specified in the range $[5,10,15,20,25,30,35] \mathrm{cm}^{-1}$. In Model 3, 


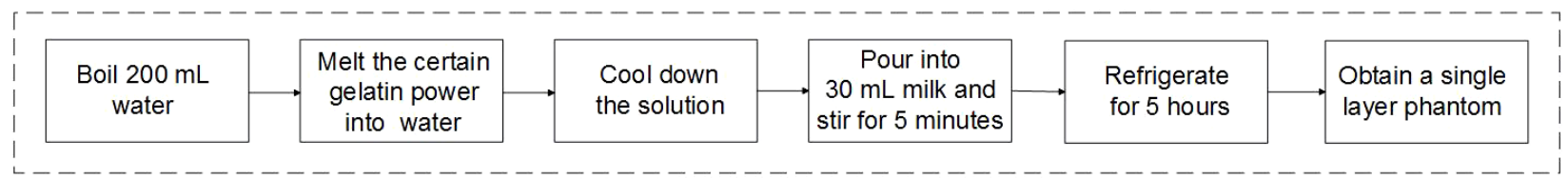

(a)

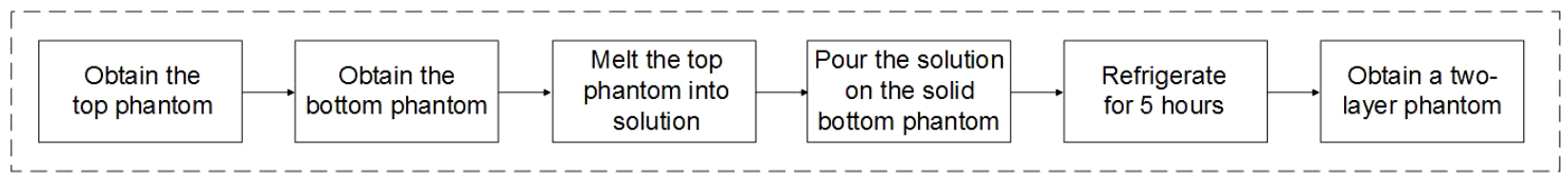

(b)

Fig. 4. The flow chart of constructing the two-layer phantom.

$\mu_{s}^{\prime}$ values of bottom phantom were specified in the range $[20,40,60,80,100,120,140] \mathrm{cm}^{-1}$.

Subsequently, the photoelectric monitoring system was used in the phantom experiments. The probe was settled on the surface of phantom model, and the reflectance light intensity was transferred into voltage by photon sensor. Then the electric signal was delivered to the computer. The data was stored in the memory.

\section{Results}

\subsection{Monte Carlo simulations}

The light intensity was obtained at the specific detecting radius of $[1.0,2.0,3.0,4.0,5.0] \mathrm{cm}$ on the tissue surface. In each model, the light intensity was normalized to the first sample.
Figure 5 illustrates the normalized light intensity changes with the increase of the CSF thickness at different detecting radius. The curves of light intensity at different detecting radius have the significant difference. With the increase of CSF thickness, the light intensity increases greatly at the detecting radius of $4.0 \mathrm{~cm}$, while the light intensity decreases little at the detecting radius of $2.0 \mathrm{~cm}$.

Figures 6(a) and 6(b) show the contour map of the light intensity whose CSF thicknesses are $0.2 \mathrm{~cm}$ and $0.8 \mathrm{~cm}$, respectively. In the CSF layer, the contour map with $0.2 \mathrm{~cm}$-thick CSF is more fluent than that of the $0.8 \mathrm{~cm}$-thick CSF, which means that CSF will reduce the spatial resolution in the optical measurement. Lin et al..$^{29}$ have reported that with the help of CSF, the photons could travel deeper and more widely in tissue, contributes to

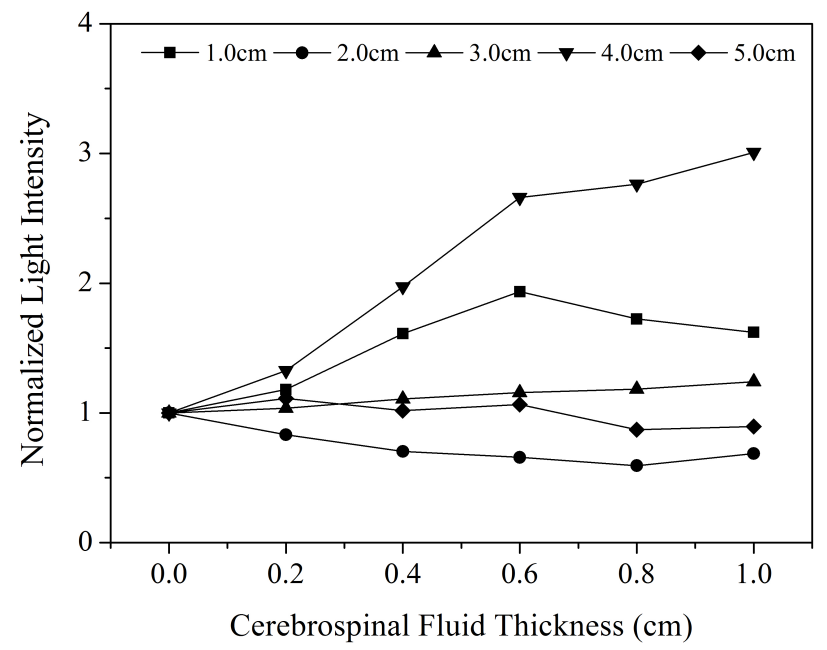

Fig. 5. Normalized light intensity with the CSF thickness at different detecting radius. 


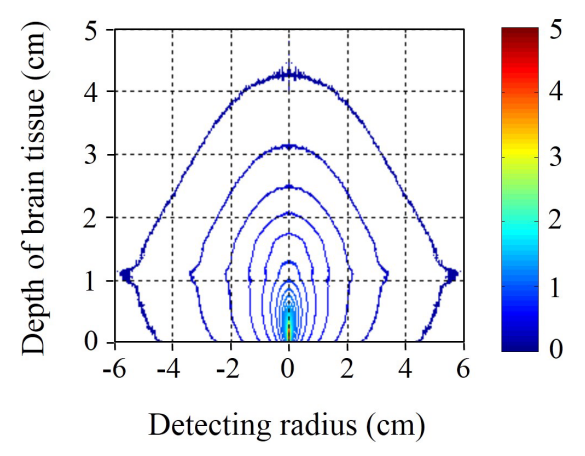

(a)

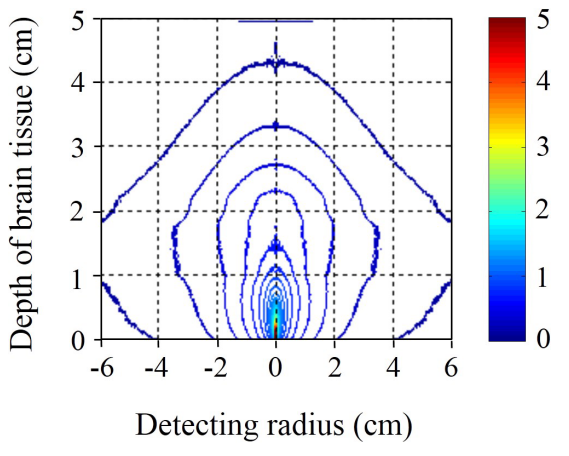

(b)

Fig. 6. Contour maps of the light intensity in the brain with different thicknesses of CSF. (a) The thickness of CSF is $0.2 \mathrm{~cm}$ and (b) is $0.8 \mathrm{~cm}$.

broader photon traces. Thus, in the light transport simulation, the CSF is an important substance that cannot be ignored.

The trends of the light intensity vs. $\mu_{s}^{\prime}$ of gray matter and white matter are shown in Fig. 7. Initial inspection of the data shows the increasing $\mu_{s}^{\prime}$ of gray matter in response to the increase of the light intensities at the detecting radius of $[2.0,3.0,4.0]$ $\mathrm{cm}$. At the detecting radius of $3.0 \mathrm{~cm}$, the light intensity has a little change with the increasing $\mu_{s}^{\prime}$ of gray matter. Figure $7(\mathrm{~b})$ shows the change of light intensity with the increasing $\mu_{s}^{\prime}$ of white matter. With the increasing $\mu_{s}^{\prime}$ of white matter, the light intensities at every detecting radius all increase.

According to the Monte Carlo simulations, the light intensity on the tissue surface was associated with the condition of brain edema and the detecting radius. If the detecting radius was $3.0 \mathrm{~cm}$ or $4.0 \mathrm{~cm}$,

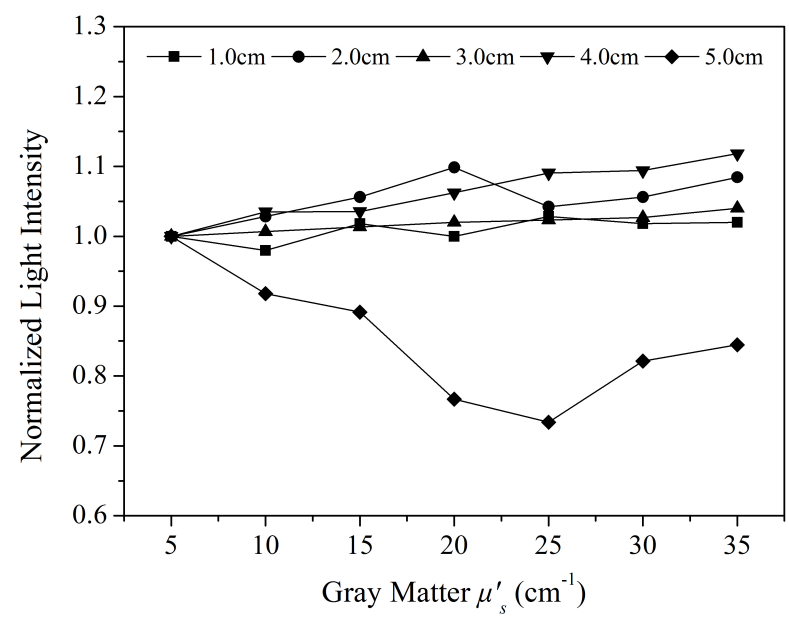

(a) the light intensity was more effectively and greatly consistent with the change of tissue and optical properties caused by brain edema. Likewise, previous studies have reported that at the detecting radius of $3.0 \mathrm{~cm}$ and $4.0 \mathrm{~cm}$, the incident light could penetrate into the deep tissue in human brain and be scattered to the surface. ${ }^{29}$

In addition, the trends of the light intensity could be totally reversed at different detecting radius when the optical properties of tissue changed. In Fig. 5, the light intensity increases in response to the increase of CSF thickness at the detecting radius of $4.0 \mathrm{~cm}$. On the other hand, the light intensity decreases vs. CSF thickness at the detecting radius of $2.0 \mathrm{~cm}$. In Fig. 7 (a), with the increasing $\mu_{s}^{\prime}$ of gray matter, the light intensity increases at the detecting radius of $4.0 \mathrm{~cm}$, while the light intensity decreases at the detecting radius of $5.0 \mathrm{~cm}$.

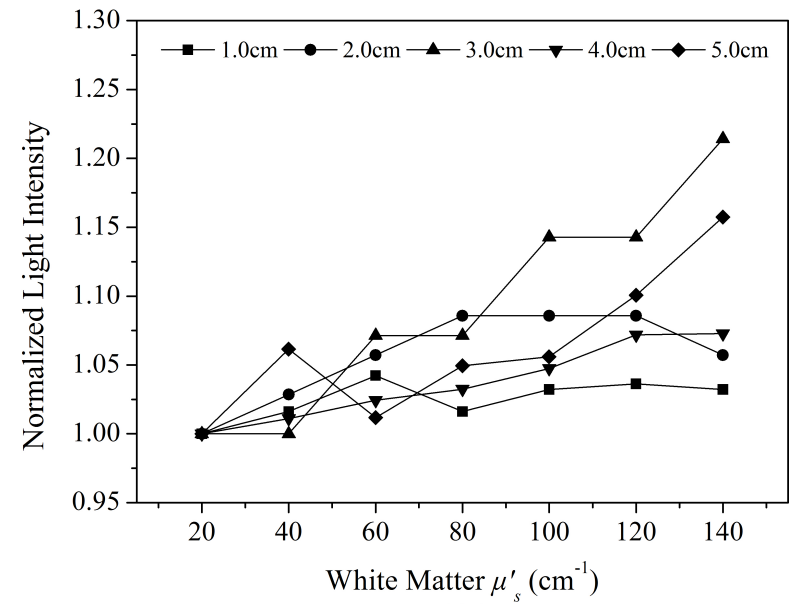

(b)

Fig. 7. Normalized light intensity with $\mu_{s}^{\prime}$ of brain substance. (a) Curves of the light intensity and $\mu_{s}^{\prime}$ of gray matter and (b) curves of the light intensity and $\mu_{s}^{\prime}$ of white matter. 


\subsection{Phantom experiments}

In the phantom experiments, $\mu_{s}^{\prime}$ and $\mu_{a}$ of each single layer phantom were calibrated by the ISS oximeter. The light intensity on the tissue surface was measured by the homemade photoelectric monitoring system.

Figure 8 shows the relationships between voltage (light intensity) and water layer thickness at the detecting radius of $3.0 \mathrm{~cm}$ and $4.0 \mathrm{~cm}$. With the increasing thickness of water layer, the voltages at the detecting radius of $3.0 \mathrm{~cm}$ and $4.0 \mathrm{~cm}$ increase, which are consistent with the results of Monte Carlo simulation. Initial inspection of the data illustrated that at the detecting radius of $3.0 \mathrm{~cm}$ and $4.0 \mathrm{~cm}$, the detectors could acquire the scattered photons (voltage).

Figure 9 shows the relationships between the voltage (light intensity) and $\mu_{s}^{\prime}$ of top phantom and bottom phantom at the detecting radius of $3.0 \mathrm{~cm}$ and $4.0 \mathrm{~cm}$. In Fig. 9(a), with the increasing $\mu_{s}^{\prime}$ of the top phantom, the voltage at the detecting radius of $3.0 \mathrm{~cm}$ has no obvious increase. The voltage at the detecting radius of $4.0 \mathrm{~cm}$ increases little, which is associated with the results in Fig. 7(a). In Fig. 9(b), the voltage at the detecting radius of $3.0 \mathrm{~cm}$ increases larger than that at the detecting radius of $4.0 \mathrm{~cm}$, which also matches the simulation results in Fig. 7(b).

The phantom experiments indicated that the voltage at the detecting radius of $3.0 \mathrm{~cm}$ was always larger than that at the detecting radius of $4.0 \mathrm{~cm}$. In each phantom model, the voltage shows the same trend with the corresponding to the simulation results. It indicated that the Monte Carlo simulation results were closed to the fact that the light traveled in the brain tissue and the results were reliable.

\section{The Combined Situations in Monte Carlo Simulation}

In clinical, different types of brain edema usually appear simultaneously, ${ }^{2}$ which means the abnormalities may occur in the CSF, gray matter and white matter simultaneously. Subsequently, we explored the combined situations of brain edema. Here, four models were presented to estimate the brain edema. Model $1^{\prime}$, Model $2^{\prime}$, Model $3^{\prime}$ and Model $4^{\prime}$ represented brain edema that occurred in the CSF and gray matter, in the CSF and white matter, in the gray matter and white matter, and in the whole brain including the CSF, gray matter and white matter.

Similar to the single situations, we obtained the same values in the combined situations. The simulation results are shown in Tables 2-5. Among them, $T_{(\mathrm{CSF})}$ means the thickness of the CSF, $\mu_{s \text { (gray matter) }}^{\prime}$ means the $\mu_{s}^{\prime}$ of gray matter, and $\mu_{s(\text { white matter })}^{\prime}$ means of the $\mu_{s}^{\prime}$ of white matter.

In Table 2, with the increase of CSF thickness and $\mu_{s}^{\prime}$ of gray matter, the light intensity decreases little at the detecting radius of $1.0 \mathrm{~cm}$ and $2.0 \mathrm{~cm}$, whereas it increases little at the detecting radius of $[3.0,4.0,5.0] \mathrm{cm}$. These change trends are similar in

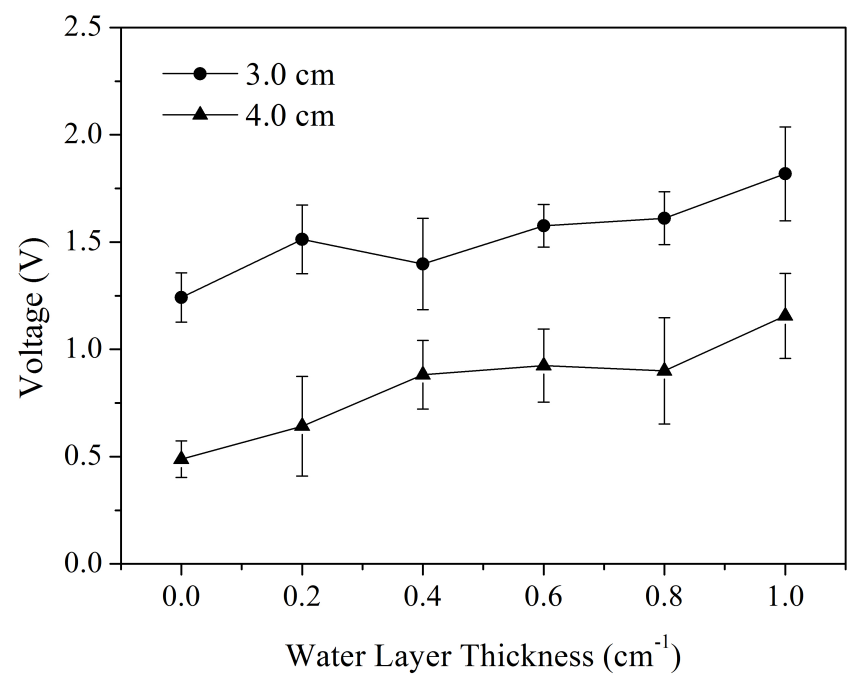

Fig. 8. Voltage with the different water layer thickness. 


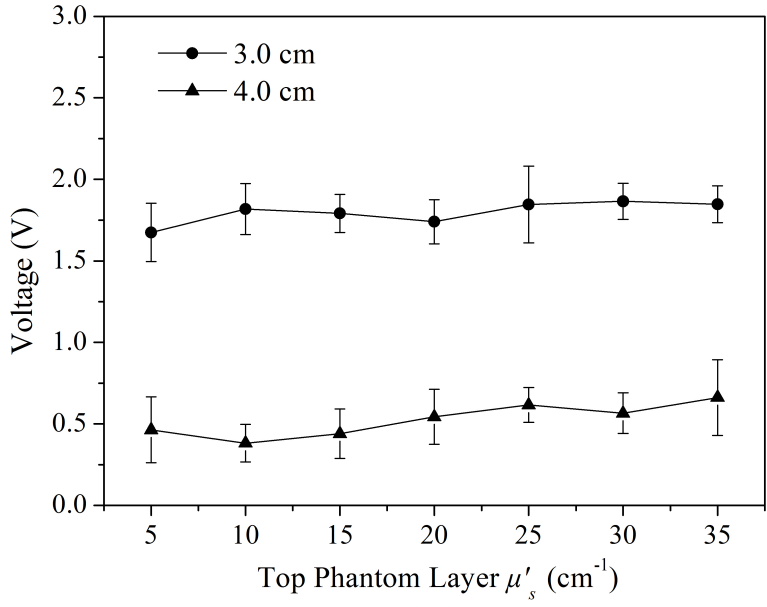

(a)

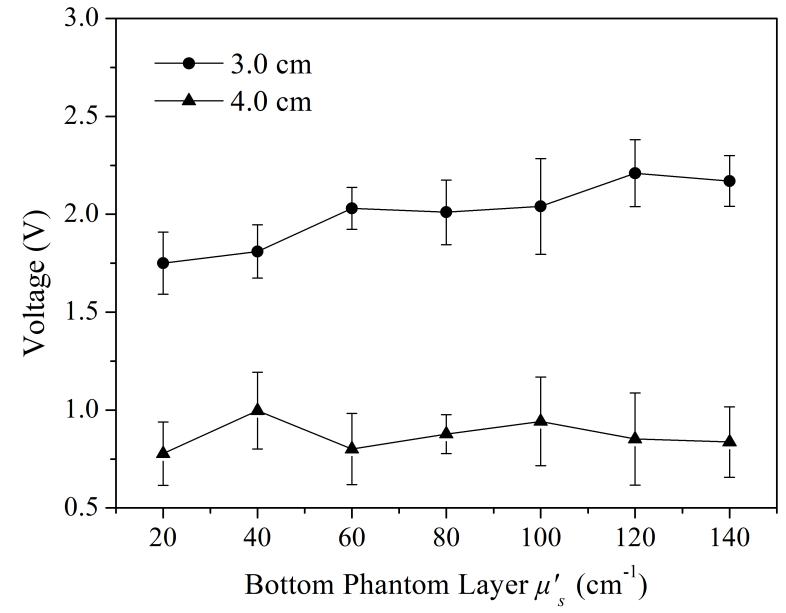

(b)

Fig. 9. Relationships of the voltage and $\mu_{s}^{\prime}$ of phantom layer. (a) Curves of the voltage and $\mu_{s}^{\prime}$ of top phantom layer and (b) curves of the voltage and $\mu_{s}^{\prime}$ of bottom phantom layer.

Tables 3 and 5. Among the detecting radiuses, the light intensity at the detecting radius of $5.0 \mathrm{~cm}$ increases most largely. In Table 4, the change trend of the light intensity is smaller than that in the other models. The light intensities, at the detecting radius of $[1.0,2.0,3.0,4.0] \mathrm{cm}$, increase with the deterioration of brain edema. Instead, the light intensity at the detecting radius of $5.0 \mathrm{~cm}$ decreases obviously. In Model 4', the light intensity at the detecting radius of $3.0 \mathrm{~cm}$ increases most largely.

When brain edema occurs in more than two layers of brain tissues, the biggest change is no longer at the detecting radius of $3.0 \mathrm{~cm}$ and $4.0 \mathrm{~cm}$. However, the light intensity still has a good relationship with optical properties at the detecting radius of $3.0 \mathrm{~cm}$ and $4.0 \mathrm{~cm}$. Taking the single situations and combined situations into consideration, the light intensity at the detecting radius of

Table 2. The normalized light intensity in Model $1^{\prime}$.

\begin{tabular}{|c|c|c|c|c|c|c|}
\hline \multicolumn{2}{|c|}{ Brain edema } & \multicolumn{5}{|c|}{ Detecting radius $(\mathrm{cm})$} \\
\hline $\begin{array}{l}\mathrm{T}_{(\mathrm{CSF})} \\
(\mathrm{cm})\end{array}$ & $\begin{array}{c}\mu_{s(\text { gray matter })}^{\prime} \\
\left(\mathrm{cm}^{-1}\right)\end{array}$ & 1.0 & 2.0 & 3.0 & 4.0 & 5.0 \\
\hline 0 & 5 & 1 & 1 & 1 & 1 & 1 \\
\hline 0.2 & 10 & 0.9889 & 0.9957 & 1.1762 & 1.3458 & 1.6564 \\
\hline 0.4 & 15 & 0.9656 & 0.9937 & 1.2750 & 1.6088 & 2.5457 \\
\hline 0.5 & 20 & 0.9633 & 0.9715 & 1.259 & 1.9149 & 3.0352 \\
\hline 0.6 & 25 & 0.9506 & 0.9506 & 1.4075 & 2.0481 & 3.6023 \\
\hline 0.8 & 30 & 0.9506 & 0.9976 & 1.4402 & 2.3660 & 4.5463 \\
\hline 1.0 & 35 & 0.9281 & 0.9283 & 1.5555 & 2.5702 & 4.9364 \\
\hline
\end{tabular}

$3.0 \mathrm{~cm}$ and $4.0 \mathrm{~cm}$ has the ability to indicate the change of optical properties in the brain tissue, which represent the situations of brain edema.

\section{Discussion}

In this study, we aimed at investigating the possibility of noninvasive optical monitoring of brain edema by NIRS. Several models were performed according to the different positions of increasing water content, such as the CSF thickness, $\mu_{s}^{\prime}$ of gray matter and white matter. The models included the single situations and combined situations. The Monte Carlo simulations and phantom experiments were designed to explore the changes of light intensity on the tissue surface. As a result, it revealed that the light intensity was related to the conditions of the brain edema as well as the detecting radius on the tissue surface. In these models, the light

Table 3. The normalized light intensity in Model $2^{\prime}$.

\begin{tabular}{|c|c|c|c|c|c|c|}
\hline \multicolumn{2}{|c|}{ Brain edema } & \multicolumn{5}{|c|}{ Detecting radius $(\mathrm{cm})$} \\
\hline $\begin{array}{l}\mathrm{T}_{(\mathrm{CSF})} \\
(\mathrm{cm})\end{array}$ & $\begin{array}{c}\mu_{s(\text { white matter })}^{\prime} \\
\quad\left(\mathrm{cm}^{-1}\right)\end{array}$ & 1.0 & 2.0 & 3.0 & 4.0 & 5.0 \\
\hline 0 & 20 & 1 & 1 & 1 & 1 & 1 \\
\hline 0.2 & 40 & 0.9859 & 1.0515 & 1.2490 & 1.6845 & 1.9081 \\
\hline 0.4 & 60 & 0.9635 & 1.0635 & 1.3735 & 2.0440 & 3.6364 \\
\hline 0.5 & 80 & 0.9644 & 1.0370 & 1.4703 & 2.3761 & 4.3877 \\
\hline 0.6 & 100 & 0.9495 & 1.0162 & 1.4651 & 2.7205 & 4.9786 \\
\hline 0.8 & 120 & 0.9467 & 1.0187 & 1.4777 & 2.9057 & 5.9310 \\
\hline 1.0 & 140 & 0.9110 & 0.9435 & 1.4624 & 3.1529 & 6.8566 \\
\hline
\end{tabular}


Table 4. The normalized light intensity in Model $3^{\prime}$.

\begin{tabular}{|c|c|c|c|c|c|c|}
\hline \multicolumn{2}{|c|}{ Brain edema } & \multicolumn{5}{|c|}{ Detecting radius $(\mathrm{cm})$} \\
\hline$\mu_{s(\text { gray matter })}^{\prime}\left(\mathrm{cm}^{-1}\right)$ & $\mu_{s(\text { white matter })}^{\prime}\left(\mathrm{cm}^{-1}\right)$ & 1.0 & 2.0 & 3.0 & 4.0 & 5.0 \\
\hline 5 & 20 & 1 & 1 & 1 & 1 & 1 \\
\hline 10 & 40 & 1.0410 & 1.0777 & 1.1136 & 1.0593 & 0.8043 \\
\hline 15 & 60 & 1.0325 & 1.1223 & 1.2020 & 1.1057 & 0.8023 \\
\hline 20 & 80 & 1.0832 & 1.1468 & 1.1938 & 1.1258 & 0.8238 \\
\hline 25 & 100 & 1.0710 & 1.1831 & 1.2629 & 1.1554 & 0.7396 \\
\hline 30 & 120 & 1.0936 & 1.2065 & 1.2878 & 1.2036 & 0.6942 \\
\hline 35 & 140 & 1.1162 & 1.1953 & 1.3338 & 1.2400 & 0.7470 \\
\hline
\end{tabular}

Table 5. The normalized light intensity in Model $4^{\prime}$.

\begin{tabular}{|c|c|c|c|c|c|c|c|}
\hline \multicolumn{3}{|c|}{ Brain edema } & \multicolumn{5}{|c|}{ Detecting radius $(\mathrm{cm})$} \\
\hline $\mathrm{T}_{(\mathrm{CSF})}(\mathrm{cm})$ & $\mu_{s(\text { gray matter })}^{\prime}\left(\mathrm{cm}^{-1}\right)$ & $\mu_{s(\text { white matter })}^{\prime}\left(\mathrm{cm}^{-1}\right)$ & 1.0 & 2.0 & 3.0 & 4.0 & 5.0 \\
\hline 0 & 5 & 20 & 1 & 1 & 1 & 1 & 1 \\
\hline 0.2 & 10 & 40 & 0.9992 & 1.0255 & 1.1784 & 1.3594 & 1.3503 \\
\hline 0.4 & 15 & 60 & 1.0245 & 1.0938 & 1.2546 & 1.7096 & 2.0431 \\
\hline 0.5 & 20 & 80 & 1.0059 & 1.1024 & 1.3499 & 1.9512 & 2.5089 \\
\hline 0.6 & 25 & 100 & 1.0091 & 1.0551 & 1.4705 & 2.1561 & 3.1480 \\
\hline 0.8 & 30 & 120 & 1.0160 & 1.0487 & 1.4267 & 2.3640 & 3.4245 \\
\hline 1.0 & 35 & 140 & 0.9792 & 1.0149 & 1.5365 & 2.5902 & 4.2819 \\
\hline
\end{tabular}

intensities at the detecting radius of $3.0 \mathrm{~cm}$ and $4.0 \mathrm{~cm}$ could effectively and greatly respond to the change of tissue parameters and optical parameters in the deep human brain tissue. With the deterioration of brain edema, the light intensities at the detecting radius of $3.0 \mathrm{~cm}$ and $4.0 \mathrm{~cm}$ show the trends of great increase. That was, no matter where the brain edema occurs, the light intensities at the detecting radius of $3.0 \mathrm{~cm}$ and $4.0 \mathrm{~cm}$ will increase. By monitoring the light intensity on the surface noninvasively, we could have a good knowledge of brain edema. However, the light intensity at the other detecting radius did not have the coincident tendency with brain edema.

The trends of the reflected light intensity will be reversed at different detecting radius when the tissue properties changes. In Fig. 7(a), with the increasing $\mu_{s}^{\prime}$ of gray matter, the light intensity increases at the detecting radius of $4.0 \mathrm{~cm}$, while the light intensity dropped at the detecting radius of $5.0 \mathrm{~cm}$. It is presumably due to the fact that with the increase of detecting radius, the transmission depth and transmission path of the incident light in the tissue has changed. Scattered by CSF and other brain tissues in different degrees, the reflectance light intensity on the tissue surface differs from each other.
Brain edema means the increase of the water content in the brain. The water content may increase in different positions, such as in the CSF, gray matter and white matter, according to different pathologies of brain edema. When the water content increases in the CSF, the increasing thickness of the CSF is the major indicator in the light transmission. When the water content increases in the gray matter and white matter, brain cells become swelling and the optical properties of brain tissue will correspondingly change, such as the reduced scattering coefficient $\left(\mu_{s}^{\prime}\right) \cdot{ }^{20,27,30}$

Because brain edema is caused by different reasons, the water content may increase in different positions, leading to different change of tissue parameters and optical properties. According to the main types of brain edema, three single situation models were established in this study. In each model, the light intensity on the tissue surface was collected to investigate the relationships between the light intensity, the detecting radius and tissue/ optical properties. Furthermore, three kinds of brain edema usually mix up in clinical. Therefore, in the Monte Carlo simulation, some combined situation models were carried out.

Based on the single situation models, the light intensity was obtained at different detecting radius 
by the Monte Carlo simulation and phantom experiments. The phantom experiment results are consistent with the Monte Carlo simulation results. Based on the combined situation models, the light intensity was explored at different detecting radius by the Monte Carlo method. Considering the difficulties of constructing all phantoms of the combined situations, as well as the verification of phantom experiments has ever affirmed in the single situation models, only the Monte Carlo simulations were performed in the combined situations.

Considering the individual differences and the difficulties of system calibration, the photoelectric monitoring system is only available to monitor the changes of brain edema, and has no access to the absolute value for assessing brain edema. When the brain edema develops, the light intensity will respond to the changes. On the other hand, if the condition of brain edema keeps stable, the light intensity also has little or no change. By observing the light intensity, we could have a good knowledge of the brain edema condition.

In this study, we did not focus on the effects of the blooding during brain edema. When patients suffer from brain edema or neurosurgical, the intracranial hemorrhage probably happens. With the bigger absorption ability than brain tissue, the massive bleeding may absorb most of incident light, which will lead to the large decrease of light intensity. The increase of the absorption of light due to blood may offset the decrease of scattering property resulting from brain edema. Thus, the change of the light intensity at the detecting radius of $3.0 \mathrm{~cm}$ and $4.0 \mathrm{~cm}$ will not be sure when the brain edema develops with the intracranial hemorrhage. In the further research, we will explore the light intensity under the intracranial hemorrhage. Meanwhile, the Monte Carlo simulations and phantom experiments are both conducted. In the Monte Carlo simulations, different positions and different areas of blood will be estimated in single situation and combined situation models. Then, an ink-mixed phantom will be constructed to estimate the blood in the phantom experiments which optical parameter is the same as human blood. The ink-mixed phantom will be also simulated in the phantom experiments.

This study only investigated the possibility of optical monitoring of the brain edema using the Monte Carlo simulations and phantom experiments. We did not conduct the animal experiments, because the research is based on the human brain and the results in the study may not be suitable for animals. Recently, we have ever produced brain edema models by injecting lipopolysaccharide (LPS) in rats. ${ }^{31}$ The optical parameters of brain tissue were detected by a homemade NIRS experimental system. It found that the $\mu_{s}^{\prime}$ of brain tissue in the edema rats could decrease by $2.5 \mathrm{~cm}^{-1}$. There were significant differences in brain edema groups (injecting $0.05 \mathrm{~mL} / 100 \mathrm{~g}$ LPS $(1 \mathrm{mg} / \mathrm{mL})$ ) compared to the blank group (no injecting) and the control group (injecting equal saline). In the future, some Monte Carlo simulations, phantom experiments as well as animal experiments may be launched based on $\mu_{s}^{\prime}$ values in the rat experiments.

\section{Conclusions}

The possibility of noninvasively monitoring of brain edema by NIRS method was explored by the Monte Carlo simulations and phantom experiments. Furthermore, many combined situations of the brain edema were also studied in this paper. It can be concluded that the light intensity was a reliable parameter to assess the brain edema, which is also influenced by the detecting radius. This paper has a potential in the medical equipment development and provides reference for medical diagnosis and treatment of the brain edema.

\section{Acknowledgments}

This work was supported by the Foundation of Graduate Innovation Center in NUAA (Grant No. kfjj20150310), National Natural Science Foundation of China (Grant Nos. 61275199 and 61378092), and the Fundamental Research Funds for the Central Universities (Grant Nos. NS2015032 and NS2015201).

\section{References}

1. R. Ahmed, B. Anish, "Medical management of cerebral edema," Neurosurg. Focus 22, 1-12 (2007).

2. S. Nag, J. Manias, D. Stewart, "Pathology and new players in the pathogenesis of brain edema," Acta Neuropathologica 118, 197-217 (2009).

3. H. Peter, T. Ivan, K. Peter, "Surgery for brain edema," Neurosurgical Focus 22, 1-9 (2007).

4. D. Liu, X. Zhang, B. Hu, B. P. Ander, "Src family kinases in brain edema after acute brain injury," Brain Edema XVI, 185-190 (2016). 
5. G. Jamshid, C. Nancy, "Intracranial-pressure monitoring in traumatic brain injury," New Eng. J. Med. 368, 1751-1752 (2013).

6. U.K. Misra, K. Jayantee, G. Gourav, "Hyperosmolar therapy for raised intracranial pressure", New Eng. J. Med. 367, 2554-2557 (2012).

7. L. Mangel, Z. Hanzély, T. Kiss, P. Agoston, "CT densitometry of the brain: A novel method for early detection and assessment of irradiation induced brain edema," Drug Testing Anal. 7, 544-549 (2015).

8. Y. Li, J. Wang, M. Li, "Quantification of brain edema and hemorrhage by MRI after experimental traumatic brain injury in rabbits predicts subsequent functional outcome," Neurol. Sci. 33, 731-740 (2012).

9. B.S.S Edson, H. Roberto, T. Jacobsen Manoel, DA Almir Ferreira, M. Raul, "Cerebral hemodynamic changes gauged by transcranial Doppler ultrasonography in patients with posttraumatic brain swelling treated by surgical decompression," J. Neurosurg. 104, 93-100 (2006).

10. Y. Su, H. Jing, Y. Guo, J. Liu, T. Liu, W. Zhang, "Application of noninvasive monitoring of intracranial pressure with flash visual evoked potential (fvep) in treatment of posttraumatic acute diffuse brain swelling (padbs) without hematoma", J. Modern Electrophysiol. 3, 1-3 (2015).

11. L. Lixu, D. Weiwei, J. Xunming, C. Lihua, C. Ling, H. Wei, "A new method of noninvasive brain-edema monitoring in stroke: Cerebral electrical impedance measurement" Neurolo. Res. 28, 31-37 (2006).

12. Y. Liu, Y. Wang, Z. Qian, "Monitoring the reduced scattering coefficient of bone tissues on the trajectory of pedicle screw placement using near-infrared spectroscopy", J. Biomed. Opt. 19, 117002 (2014).

13. V. R. Kondepati, H. M. Heise, J. Backhaus, "Recent applications of near-infrared spectroscopy in cancer diagnosis and therapy," Anal. Bioanal. Chem. 390, 125-139 (2007).

14. Eri Otsukaa, Hiroyuki Abea, Masaki Aburadaa, Makoto Otsuka, "Nondestructive prediction of the drug content of an aspirin suppository by near-infrared spectroscopy," Drug Develop. Indust. Pharm. 36, 839-844 (2010).

15. Z. Qian, Sundar Victor, Y. Gu, Cole Giller, H. Liu, "'Look-Ahead Distance' of a fiber probe used to assist neurosurgery: Phantom and Monte Carlo study," Opt. Exp. 11, 1844-1855 (2003).

16. Izumi Nishidate, Keiichiro Yoshida, Satoko Kawauchi, Shunichi Sato, Manabu Sato, "In vivo estimation of light scattering and absorption properties of rat brain using a single-reflectance fiber probe during cortical spreading depression," J. Biomed. Opt. 20, 1-13 (2015).

17. B. Chance, H. Liu, T. Kitai, Y. Zhang, "Effects of solutes on optical properties of biological materials:
Models, cells, and tissues," Anal. Biochemi. 227, 351-362 (1995).

18. J. Xie, Z. Qian, T. Yang, W. Li, G. Hu, "Near-infrared spectroscopy technique to evaluate the effects of drugs in treating traumatic brain edema," The 9th Int. Conf. Photonics and Imaging in Biology and Medicine, Nanjing, Jiangsu (2011).

19. Harold K. Kimelberg, "Current concepts of brain edema," J. Neurosurg. Publishing Group 83, 10511059 (2009).

20. J. Xie, Z. Qian, T. Yang, W. Li, G. Hu, "Minimally invasive assessment of the effect of mannitol and hypertonic saline therapy on traumatic brain edema using measurements of reduced scattering coefficient $\left(\mu^{\prime} s\right), "$ Appl. Opt. 49, 5407-5414 (2010).

21. L. Dai, G. Hua, Z. Qian, "Application of near infrared diffusion spectrum in effect of anhydration solution," Chin. J. Quantum Electron. 27, 737-742 (2010).

22. L. Wang, SL. Jacques, "Hybrid model of Monte Carlo simulation diffusion theory for light reflectance by turbid media," J. Opt. Soc. Am. A 10, 1746-1752 (1993).

23. L. Wang, S.L. Jacques, L. Zheng, "MCML-Monte Carlo modeling of light transport in multi-layered tissues," Comput. Meth. Prog. Biomed. 47, 131-146 (1995).

24. S Umeyama, T. Yamada, "Monte Carlo study of global interference cancellation by multidistance measurement of near-infrared spectroscopy," J. Biomed. Opt. 14, 717-722 (2009).

25. N. Fravi, "Brain edema," Therapeutische Umschau Revue Thérapeutique 61, 679-686 (2004).

26. J. Xie, Z. Qian, N. Deng, T. Yang, W. Li, G. Hu, "Monitoring traumatic brain injury by using functional near-infrared spectroscopy," Chin. J. Lasers 38, 104004 (2011).

27. I. Nishidate, C. Mizushima, K. Yoshida et al. " In vivo estimation of light scattering and absorption properties of rat brain using a single-reflectance fiber probe during cortical spreading depression", $J$. Biomedi. Opt. 20, 1-13 (2015).

28. A. H. Hielscher, H. Liu, B. Chance, FK Tittel, S.L. Jacques, "Time Resolved Photon Emission from Layered Turbid Media," Appl. Opt. 36, 719-728 (1996).

29. Fang Lin, H. Ding, Feng Wang, Chang Su, "Multilayer Monte-Carlo simulation for non-invasive nearinfrared measurement on head," J. Optoelectron. Lasers 10, 1-8 (1999).

30. L. He, Y. You, T. Yang et al. "Study on mininvasive monitoring of traumatic brain edema in vivo by near-infrared spectroscopy," Mod. Medi. J. 36, 221-224 (2008).

31. H. Wang, J. Lu, Q. Luo et al., "The roles of TNF- $\alpha$ and IL-8 in the pathogenesis of brain edema induced by LPS in rat," Chi. J. Immunol. 19, 60-62 (2003). 